\title{
PROTOCOLO DE CONTROLE DA DOR E CANSA- ÇO POR MEIO DE EXERCÍCIOS NA FIBROMIAL- GIA: UMA NOVA PROPOSTA
}

\author{
Fernando Schorr Grossl', Marcos Brioschiं ${ }^{2}$, Renato Vilella ${ }^{3}$, Clodoaldo Antônio De Sá ${ }^{4}$ \\ 1 Programa de Pós-Graduação em Ciências da Saúde, Universidade Comunitária da Região de Chapecó. \\ ${ }^{2}$ Coordenador da Especialização em Termologia e Termografia Médica do HCFMUSP. \\ 3 Pesquisador autônomo. Belo Horizonte, Minas Gerais. \\ ${ }_{4}^{4}$ Programa de Pós-Graduação em Ciências da Saúde, Universidade Comunitária da Região de Chapecó
}

Resumo: Embora a ciência tenha avançado na terapia da fibromialgia, o cotidiano do tratamento clínico ainda é bastante desafiador. Diante disso, o objetivo desse estudo é apresentar um novo protocolo de exercícios fáceis de aplicar, baseado no controle da dor e cansaço durante as intervenções por meio da percepção subjetiva de uma paciente com fibromialgia. Resultado: A nova proposta consistiu de exercícios físicos associados à percepção subjetiva de dor e cansaço para o controle da intensidade e volume dos exercícios. As avaliações ocorreram por meio da escala visual analógica para dor (EVA) e da escala de percepção subjetiva de esforço (BORG). As análises de dor e cansaço foram feitas antes, durante e logo após cada sessão. A intervenção teve duração de 24 semanas com frequência de duas vezes por semana. Após a avaliação inicial, a partir do diagnóstico, foi dado início ao protocolo de treinamento de força para todos os grupos musculares. Conclusão: $\mathrm{O}$ uso das variáveis dor e cansaço para determinar a intensidade e volume durante o protocolo de exercícios, resultou na melhora do quadro doloroso ao final da intervenção. Resultado que se manteve após seis meses da alta clínica. Permitindo a retomada da prática regular de exercícios físicos.

\section{Palavras-chave: Fibromialgia, Dor crônica, Reabilitação, Exercício}

Abstract: Although science has advanced in the therapy of fibromyalgia, the daily routine of clinical treatment is still quite challenging. Therefore, the objective of this study is to present a new protocol of exercises that are easy to apply, based on pain and tiredness control during interventions through the subjective perception of a patient with fibromyalgia. Result: The new proposal consisted of physical exercises associated with subjective perception of pain and tiredness to control the intensity and volume of the exercises. Evaluations were performed using the visual analog scale for pain (VAS) and the subjective perception of effort scale (BORG). Pain and tiredness analyses were performed before, during, and shortly after each session. The intervention lasted 24 weeks with frequency twice a week. After the initial evaluation, from the diagnosis, the strength training protocol was initiated for all muscle groups. Conclusion: The use of the variables pain and tiredness to determine the intensity and volume during the exercise protocol resulted in the improvement of the painful condition at the end of the intervention. Result that remained after six months of clinical discharge. Allowing the resumption of the regular practice of physical exercises.

Keywords: Fibromyalgia, Chronic pain, Therapies, Exercise. 
$\vdash$ PAJMT

\section{INTRODUÇÃO}

A fibromialgia (FM) é uma condição clínica caracterizada por dor crônica generalizada, alteração no limiar de dor, fadiga e perda funcional, distúrbios de sono e comprometimento cognitivo, decorrente de um conjunto de alterações resultantes de interações periféricas e centrais ${ }^{1}$. Segundo a literatura, a FM tem prevalência entre 0,2 a $6,6 \%$ na população em geral, sendo mais comum em mulheres $(2,4 \text { a 6,8\% })^{2}$.

No Brasil, a FM acomete cerca de $2 \%$ da população total ${ }^{3}$. Além da condição clínica característica, a FM acarreta distúrbios psicológicos como instabilidade emocional, dificuldade para se concentrar no trabalho, realizar tarefas de vida diária e cultivar relacionamentos, levando a paciente ao isolamento social, condições que, em conjunto, impactam negativamente na saúde e qualidade de vida ${ }^{4}$.

Por envolver uma condição clínica e biopsicossocial complexa, a adesão constitui uma tarefa difícil e requer abordagens que busquem aliar estratégias e terapias de aderência ao tratamento ${ }^{5-8}$.

Nesse contexto, com objetivo de avaliar o efeito do controle da dor e cansaço durante as intervenções com exercícios físicos na fibromialgia este trabalho apresenta uma nova proposta de tratamento, baseado em um protocolo de exercícios físicos conduzidos com controle e manejo da intensidade de dor e cansaço, por meio da escala visual analógica de dor (EVA) e a escala de percepção subjetiva de esforço $(\mathrm{BORG})^{7-9}$

\section{METOdOLOGIA}

A nova proposta foi aplicada em uma mulher de 43 anos com diagnóstico de fibromialgia sem melhora com métodos tradicionais de tratamento. Tinha histórico de dor e contratura muscular em todo o corpo, há mais de 13 anos, principalmen- te na região torácica. A paciente relatava crises de dores que a faziam perder o controle das pernas e, por vezes, sentir medo de perder a consciência durante a crise. A dor era do tipo queimação, que se espaIhava por todo o corpo. Apresentou perda dos sentidos inúmeras vezes por causa da dor intensa. Na avaliação psicológica, foi caracterizado transtorno depressivo recorrente com episódio atual grave sem sintomas psicóticos segundo Classificação Internacional de Doenças (CID-10), (F.33.2). Passou por acompanhamento psiquiátrico e psicológico, fez uso de relaxantes musculares, analgésicos, antidepressivos, opioides, anti-inflamatórios, sem melhora satisfatória da dor.

Há 3 anos foi fechado diagnóstico de fibromialgia de acordo com a classificação e critérios da American College of Rheumatology (ACR 1990-2010), sendo encaminhada para realização do exame de termografia cutânea de corpo total, segundo protocolo da Associação Brasileira de Termologia ${ }^{10}$, ilustrado na Figura 1.

O exame registrou alterações neurovegetativas comuns a síndrome fibromiálgica como, sinal do manto térmico (distúrbio termorregulatório), vasoespasmo em extremidades (hiperatividade adrenérgica), congestão periocular e sinais de disfunção gastrointestinal. Além de disfunção miofascial de suboccipitais e trapézio superior bilateral; extensores do carpo da mão direita e multifocal de glúteo esquerdo.

$\mathrm{Na}$ avaliação dos autores (FSG, RV, CAS) foram confirmados também pontos de dor nas regiões cervicais, cintura escapular, torácica, lombar, ombros e quadril, totalizando 16 pontos miofasciais de dor, com intensidade 9/10 pontos pela Escala Visual Analógica (EVA), diminuição da força muscular (grau IV) e sinal de Godet positivo na região torácica. Segundo Classificação Internacional de Funcionalidade, 


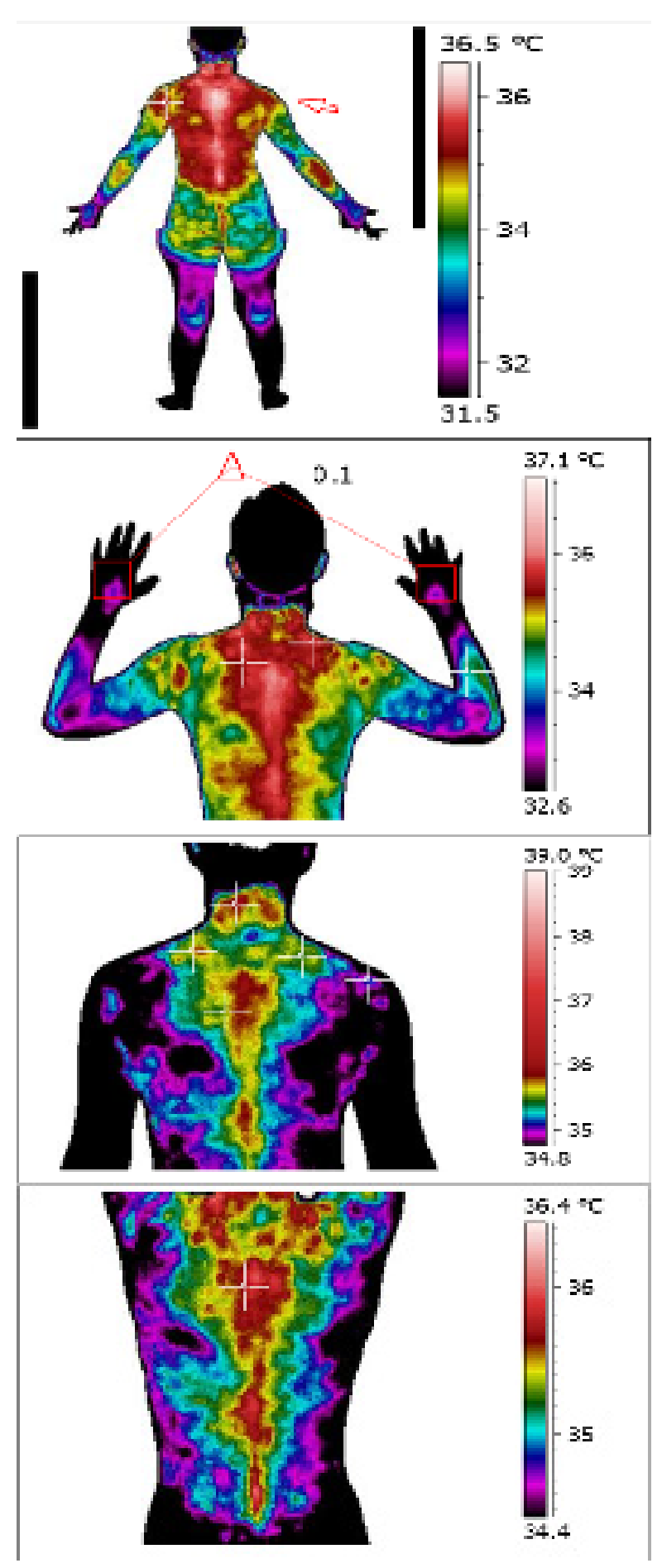

Figuura 1 - Termografia cutânea de corpo total. Documentação das alterações neurovegetativas e miofasciais na paciente com fibromialgia.

Incapacidade e Saúde (CIF) a paciente foi compatível com o descritor b280 e qualificador (d): dor limitante ou dificuldade grave, isto é, presente em mais de $50 \%$ do tempo, com uma intensidade que prejudica/rompe parcialmente a vida diária e que ocorreu frequentemente nos últimos 30 dias.
Em seguida foi iniciado o protocolo de exercícios que foram executados conforme a percepção de dor e cansaço da paciente. Antes do início dos exercícios, a paciente era questionada sobre a sua dor e cansaço, sendo atribuído uma nota entre 0 a 10. Durante a execução dos exercícios, a nota atribuída pré-intervenção, não poderia ultrapassar dois pontos. Se a nota para dor ou cansaço fosse 6/10, o critério para interromper a intervenção era o relato de aumento da dor ou cansaço superior a oito, ou seja, tinha que manter uma variação de no máximo dois pontos acima da nota atribuída pré-intervenção.

O protocolo incluiu exercícios contra resistência e exercícios aeróbicos realizados durante seis meses com uma frequência de duas vezes por semana, num total de 48 sessões, conforme descrito a seguir:

- Aquecimento na bicicleta horizontal por cinco a dez minutos, com intensidade três em cinco;

- Três séries de 10 repetições para o exercício de agachamento "sentar/ levantar", seguidos de flexão e extensão no leg press com carga dentro da análise subjetiva da dor e as repetições dentro da análise subjetiva do cansaço, que, para a carga, variou entre 30 e 40\% de 1RM;

- Aquecimento em cicloergômetro de braço por cinco minutos, intensidade três em cinco;

- Três séries de 10 repetições da puxada unilateral na posição ortostática no aparelho Cross Over, seguido de um exercício de empurrar unilateral na mesma posição ortostática e no mesmo aparelho Cross Over com carga entre $20 \%$ e $40 \%$ de $1 \mathrm{RM}$, estabelecido dentro da análise de dor subjetiva e cansaço subjetivo, respectivamente. 


\section{RESULTADOS}

O principal desfecho deste relato de caso foi a remissão total da dor em 24 semanas de intervenção (Figura 2). Nas primeiras 12 semanas, a dor reduziu em $55,6 \%$ e a redução média da dor ao longo do tratamento foi de $4,2 \%$ por semana.

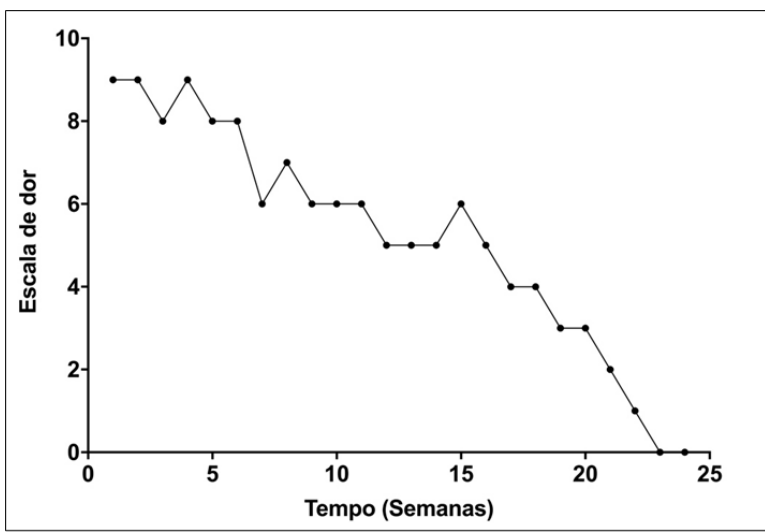

Figura 2 - Evolução da dor ao longo das 24 semanas de intervenção utilizando a Escala Visual Analógica.

\section{Discussão}

De acordo com a literatura os exercícios físicos são a linha de frente para o tratamento da dor crônica ${ }^{11-13}$. Entretanto, não existe consenso quanto a carga, volume ou frequência ideal para cada caso de dor crônica, em especial na fibromialgia $4,7,11$. A proposta de intervenção neste relato de caso, teve como principal objetivo a meIhorar da dor. Sendo o segundo objetivo, demonstrar que por meio do equilíbrio da intensidade e volume, durante a execução dos exercícios, é possível regular os efeitos inibitórios e excitatórios no sistema nervoso central da dor, usando a EVA e a BORG.

São várias as barreiras que dificultam o tratamento do paciente com fibromialgia, insuficiência no controle da dor, medo dos efeitos colaterais, falta de esclarecimento dos profissionais, além do sofrimento do julgamento alheio. A importância deste protocolo terapêutico se dá por se tratar de uma intervenção fácil e de baixo custo, com maior controle para possíveis sintomas de piora do quadro de dor. Isso pode diminuir as chances de abandono ao tratamento.

A estratégia dos autores utilizando o controle de dor e cansaço, baseados na percepção subjetiva da paciente, permitiu conduzir o tratamento até a sua alta clínica, mantendo a paciente em atividade regular de exercícios físicos mesmo após o tratamento. Do contrário, uma condução fatigante de uma única sessão de exercício, poderia aumentar o quadro de dor por sensibilizar receptores N-metil-D-aspartato (NMDA) na medula rostral ventromedial do bulbo (RVM), o que levaria ao abandono do tratamento, sugerindo uma facilitação da dor induzida pelo exercício ${ }^{13-15}$. Já a manutenção da prática regular deste protocolo de exercícios físicos dentro do controle de dor e cansaço, parece ter sido suficiente para promover a melhora do quadro de dor, sugerindo uma redução da ativação dos receptores (NMDA), diminuindo a sensibilização central ${ }^{13,14,16}$.

Além disso, a prática regular de exercícios físicos, ajuda a reduzir a expressão de transportadores de serotonina, aumentando os seus níveis no sistema nervoso central. Também, aumenta os níveis de opioides endógenos na substância cinzenta periaquedutal e na região rostroventral medial (RVM), sugerindo uma ativação endógena do sistema modulatório descendente ${ }^{13,14,16,17}$

A abordagem deste protocolo utilizando duas variáveis fisiológicas, dor e cansaço, parece ser uma boa estratégia para o controle da intensidade e volume de exercícios terapêuticos e até mesmo para as atividades de vida diária. Podendo ser efetivo na melhora da queixa dolorosa e ajudando na adesão ao tratamento. Isso porque, na literatura atual não existe consenso para prescrever intensidade e volume de exercícios em condições de dor crônica como na fibromialgia ${ }^{7,17}$.

O resultado deste caso demonstra a re- 
levância clínica do controle da intensidade e volume dos exercícios físicos, por meio do controle da intensidade da dor e cansaço. Possibilitando, um protocolo individualizado e efetivo para a melhora da dor. Além de aumentar as chances de continuidade do tratamento, tão importante para manter a abordagem centrada no acolhimento e nas várias consequências da dor e de encontro com a literatura ao definir uma abordagem terapêutica em favor do paciente $e^{6,15}$.

\section{CONCLUSÃo}

O uso deste protocolo considerando dor e cansaço para determinar a intensidade e volume durante o protocolo de exercícios, resultou na melhora do quadro doloroso ao final da intervenção. Resultado que se manteve após seis meses da alta clínica, permitindo a retomada da prática regular de exercícios físicos.

\section{AgRAdeCIMENTOS}

Professor Clodoaldo Antônio De Sá - Programa de Pós-Graduação em Ciências da Saúde, Universidade Comunitária da Região de Chapecó. Professor Marcos Brioschi - Coordenador da Especialização em Termologia e Termografia Médica do HCFMUSP

\section{REFERÊNCIAS}

1. Belt, N. K., Kronholm, E., \& Kauppi, M. J. (2009). Sleep problems in fibromyalgia and rheumatoid arthritis compared with the general population. Clinical \& Experimental Rheumatology, 27(1), 35.

2. Marques, A. P., do Espírito Santo, A. D. S., Berssaneti, A. A., Matsutani, L. A., \& Yuan, S. L. K. (2017). Prevalence of fibromyalgia: literature review update. Revista Brasileira de Reumatologia (English Edition), 57(4), 356-363. doi: 10.1016/j.rbre.2017.01.005

3. Heymann, R. E., Paiva, E. S., Martinez, J. E., Helfenstein Jr, M., Rezende, M. C., Provenza, J. R., ... \& Souza, E. J. (2017). Novas diretrizes para o diagnóstico da fibromialgia. Revista Brasileira de Reumatologia, 57, 467-476. doi: http://dx.doi.org/10.1016/j.rbr.2017.05.006

4. Meulders, A., Boddez, Y., Blanco, F., Van Den Houte, M., \& Vlaeyen, J. W. (2018). Reduced selective learning in patients with fibromyalgia vs healthy controls. Pain, 159(7), 1268-1276. doi: 10.1097/j.pain.0000000000001207

5. Wolfe, F., Clauw, D. J., Fitzcharles, M. A., Goldenberg, D. L., Katz, R. S., Mease, P., ... \& Yunus, M. B. (2010). The American College of Rheumatology preliminary diagnostic criteria for fibromyalgia and measurement of symptom severity. Arthritis Care \& Research, 62(5), 600-610. doi: 10.1002/acr.20140.

6. Løge-Hagen, J. S., Sæle, A., Juhl, C., Bech, P., Stenager, E., \& Mellentin, A. I. (2018). Prevalence of depressive disorder among patients with fibromyalgia: systematic review and meta-analysis. Journal of Affective Disorders, 245, 1098-1105. doi: 10.1016/j.jad.2018.12.001.

7. Gatzounis, R., Schrooten, M. G., Crombez, G., \& Vlaeyen, J. W. (2012). Operant learning theory in pain and chronic pain rehabilitation. Current pain and headache reports, 16(2), 117-126. doi: 10.1007/s11916-012-0247-1.

8. Grayston, R., Czanner, G., Elhadd, K., Goebel, A., Frank, B., Üçeyler, N., ... \& Alam, U. (2019, April). A systematic review and meta-analysis of the prevalence of small fiber pathology in fibromyalgia: Implications for a new paradigm in fibromyalgia etiopathogenesis. In Seminars in arthritis and rheumatism (Vol. 48, No. 5, pp. 933-940). WB Saunders. doi: 10.1016/j.semarthrit.2018.08.003.

9. Martinez, J. E., Grassi, D. C., \& Marques, L. G. (2011). Análise da aplicabilidade de três instrumentos de avaliação de dor em distintas unidades de atendimento: ambulatório, enfermaria e urgência. Revista Brasileira de Reumatologia, 51(4), 299-308. https://doi.org/10.1590/ S0482-50042011000400002

10. Brioschi ML, Macedo JF, Macedo RAC. Termo-metria cutânea: novos conceitos. J Vasc Bras. 2003;2:151-60.

11. Romeyke, T., Noehammer, E., \& Stummer, H. (2018). Interdisciplinary assessment-oriented treatment of fibromyalgia: a case report. Integrative medicine research, 7(2), 200-205. doi: 10.1016/j.imr.2018.02.002

12. Plesner, K. B., \& Vaegter, H. B. (2018). Symptoms of fibromyalgia according to the 2016 revised fibromyalgia criteria in chronic pain patients referred to multidisciplinary pain rehabilitation: influence on clinical and experimental pain sensitivity. The Journal of Pain, 19(7), 777-786. doi: 10.1016/j.jpain.2018.02.009.

13. Lima, L. V., Abner, T. S., \& Sluka, K. A. (2017). Does exercise increase or decrease pain? Central mechanisms underlying these two phenomena. The Journal of physiology, 595(13), 4141-4150.

14. Bement, M. K. H., \& Sluka, K. A. (2016). Exerciseinduced analgesia: an evidence-based review. Mechanisms and Management of Pain for the Physical Therapist, 10, 177-201. doi: 10.1097/j.pain.0000000000001235.

15. Kroll, H. R. (2015). Exercise therapy for chronic pain. Physical Medicine and Rehabilitation Clinics, 26(2), 263281. doi: 10.1016/j.pmr.2014.12.007. 


\section{PAN AMERICAN JOURNAL OF MEDICAL THERMOLOGY}

16. Louw, A., Zimney, K., O'Hotto, C., \& Hilton, S. (2016).

—РAjмт The clinical application of teaching people about pain. Physiotherapy theory and practice, 32(5), 385-395. doi: 10.1080/09593985.2016.1194652.

17. Ligato, D., Petersen, K. K., Mørch, C. D., \& ArendtNielsen, L. (2018). Offset analgesia: the role of peripheral and central mechanisms. European Journal of Pain, 22(1), 142-149. doi: 10.1002/ejp. 1110. 